\title{
Integration of OWL Ontologies in MPEG-7 and TV-Anytime Compliant Semantic Indexing
}

\author{
Chrisa Tsinaraki, Panagiotis Polydoros, and Stavros Christodoulakis
}

Technical University of Crete, Laboratory of Distributed Multimedia Information Systems and Applications (TUC/MUSIC), University Campus, 73100 Kounoupidiana, Crete, Greece

\{chrisa,panpolyd, stavros\}@ced.tuc.gr

\begin{abstract}
We describe a systematic methodology for extending the audiovisual content description standards (MPEG-7 and TV-Anytime) with domain-specific knowledge descriptions expressed in OWL. The domain-specific descriptions of the audiovisual content metadata are completely transparent to applications and tools that use MPEG-7 and TV-Anytime, allowing them to use the domainspecific ontologies without any software changes. We also present an interoperability mechanism between OWL and the audiovisual content description standards, which allows MPEG-7 and TV-Anytime descriptions and their domain-specific extensions to be described in OWL and vice versa. Thus, the methodology and the mechanisms presented here open up opportunities for reusing ontology tools and ontologies across a large number of applications (with or without audiovisual content) and across different professional and user communities. We present the details of the methodology and the implementation of the tools supporting it as well as its integration in a large framework for domain-specific indexing and retrieval of audiovisual content.
\end{abstract}

\section{Introduction}

During the last few years the provision of content services in specialized environments (e.g. digital libraries, web applications, digital TV applications, m-commerce, tourism, e-learning etc.) increases very rapidly. Emphasis is given in the provision of advanced personalized services, the final form of which is specified by the service provision context.

The success of such services imposes the support of advanced methods for the retrieval of audiovisual material, based on the material content. These methods may be used directly by the end-user requests (e.g. by queries for the retrieval of soccer games where a certain player scores) or transparently to him/her, by the services (s)he invokes (e.g. presentation of news items that are of user interest according to his/her preference profile).

Advanced audiovisual content retrieval methods require the existence of contentdescription metadata for the material. Several standards have been developed for the representation of metadata for audiovisual content description, among which the dominant ones are MPEG-7 [11] and TV-Anytime [12]. MPEG-7 provides in the Semantic Part of the MPEG-7 MDS [6] the complex data types needed for the full semantic description of audiovisual content as a set of Description Schemes (DSs). 
TV-Anytime on the other hand provides only keyword-based capabilities for the semantic description of audiovisual content.

The major shortcoming of implementing systems based on one of the dominant standards [8] [7] [9] is that both MPEG-7 and TV-Anytime provide general-purpose structures for metadata representation, but not a concrete methodology for domainspecific extensions. Domain-specific knowledge however, could greatly increase the retrieval effectiveness of audiovisual content retrieval and it could also improve the user interaction with the system. In addition, there are no methodologies and tools currently which allow domain-specific ontologies defined in a general purpose ontology description language like OWL to be used with the audiovisual content description standards like MPEG-7 and TV-Anytime.

We have recently proposed the DS-MIRF (Domain-Specific Multimedia Indexing, Retrieval and Filtering) framework for the extension of MPEG-7 with domainspecific ontologies in a way that is transparent to applications that use MPEG-7 [14] [17]. This way retrieval effectiveness for multimedia content in specific application domains is enhanced. In addition, user interaction for query or profile specification is benefited from the use of the ontologies. This essentially provides a methodology for MPEG-7 to be used as an ontology specification language, for ontologies that are relevant to and can be expressed in MPEG-7.

There is however a major issue of interoperability with other ontology specification languages. Recently, OWL [3] has become the dominant standardization effort of the international research community in this area. This implies that in the future we will see many ontologies in specific knowledge domains expressed in OWL. It will be certainly easier for interoperability across applications (including audiovisual applications and applications that are not necessarily audiovisual) to have ontologies described in a standard ontology language. In such an environment it will be possible to extract (parts of) ontologies, join ontologies, map ontologies, import ontologies to different applications etc. It is of crucial importance therefore to be able to define MPEG-7 compatible ontologies in OWL and map them to MPEG-7 as well as vice versa, to transform ontologies expressed in MPEG-7 into ontologies expressed in OWL.

In this paper, we describe a methodology for the integration of OWL ontologies in the DS-MIRF framework that we have developed for the support of ontology-based semantic indexing and retrieval of audiovisual content, which follows the MPEG-7, and TV-Anytime standard specifications for metadata descriptions. Such methodologies have not been described in the literature so far.

An essential part of this methodology is to develop in OWL an ontology that fully captures the semantic metadata model defined in the Semantic Part of the MPEG-7 MDS. The need for an ontology that fully captures the MPEG-7 metadata model has been pointed out by several research groups [1] [13] [5]. Some important work in this direction has been carried out in [4] for the case of Resource Description Framework (RDF) [10], but it has some limitations: Classes corresponding to the MPEG-7 complex types have been defined, but not all of the (simple and complex) attributes of the classes are represented. In addition, typed relationships among the metadata items are not represented, although MPEG-7 provides complete support for typed relationships. Furthermore, this work has been based on RDF, which suffers form several limitations for representing ontologies, while the dominant standardization effort in ontology representation is now OWL. 
The work described in this paper provides a systematic methodology for extending the multimedia content description standards with domain-specific knowledge descriptions expressed in OWL, and creates an interoperability mechanism between OWL and the dominant multimedia content description standards (MPEG-7 and TVAnytime), thus opening up opportunities for reusing ontology tools and ontologies in a large number of applications and professional and user communities.

The rest of the paper is organized as follows: A brief overview of the DS-MIRF framework architecture focusing on the modules implementing OWL ontology integration is provided in section 2. The methodology of OWL ontology integration in the framework is discussed in section 3. The core ontology is described in section 4, while the methodology for domain-specific ontology definition is discussed in section 5. The transformation rules for the production of MPEG-7 and TV-Anytime compliant metadata from the RDF metadata structured according to the core ontology and the domain-specific ontologies are described in section 6. Conclusions and future work are discussed in section 7.

\section{Overview of the DS-MIRF Framework}

In this section we provide an overview of the architecture of the DS-MIRF framework that supports ontology-based semantic indexing and retrieval of audiovisual content, with emphasis in the framework modules where OWL ontology integration takes place. The framework architecture is depicted in Fig. 1.

As shown in Fig. 1, OWL ontologies are present during the Segmentation and Semantic Indexing process, when they are imported in the system in a systematic way. The use of the ontologies enhances the retrieval effectiveness but does not affect the software that uses MPEG-7 and TV-Anytime constructs only. This is achieved through the provision of appropriate search APIs and compatible semantic indexing for both standards. The major issue in our approach is the provision of end-user search interfaces based on both MPEG-7 and TV-Anytime standard specifications. This way, search and profiling software developed by third-parties that is compatible to MPEG-7 and TV-Anytime can still be used to access the audiovisual content. The major components of the framework architecture are:

- The Segmentation \& Semantic Indexing Tool, used during the audiovisual content segmentation process, which also includes semantic indexing. Semantic indexing is carried out using the Semantic Indexing Component of the tool, which is responsible for the import of domain-specific OWL ontologies and application-specific $R D F$ metadata (in both cases whenever needed), the definition of both application specific metadata and instance description metadata, and their storage in the system database. For example, in a soccer tournament application the applicationspecific metadata may be instances of the teams that participate in the tournament, instances of the team players, instances of the referees, coaches etc. These instances are reusable both in one game (e.g. they can be related with several events in the video program of the specific game), as well as across several games in the tournament. The instance description metadata describe in general which events of the real world take place in which parts of the video. The event types, the participants of the events, the time and the place that the events take place are described 
by the ontologies and the application-specific metadata. During the segmentation, MPEG-7 compliant semantic metadata as well as TV-Anytime metadata (keywords), which are validated against the ontologies and the application-specific metadata are produced (through the transformation of the RDF metadata formed according to the ontologies to metadata compliant to MPEG-7 and TV-Anytime) and used for the indexing of programs and segments. During querying or user profile formation, the specific queries or the user profile are validated against the ontologies and the application-specific metadata.

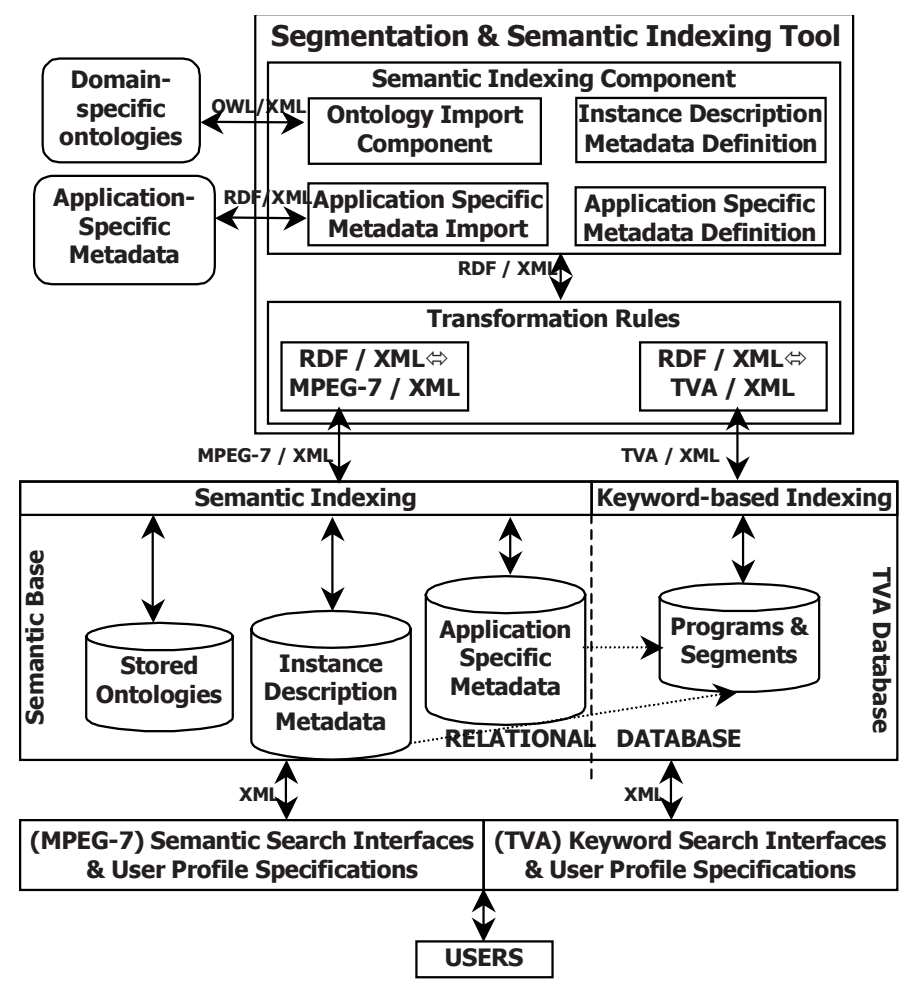

Fig. 1. Architectural Overview of the DS-MIRF Framework

- A relational database, where audiovisual segments and the metadata that describe them are stored. The relational database contains an MPEG-7 compliant Semantic Base, where the semantic metadata are stored and a TV-Anytime compliant Database where TV-Anytime metadata for audiovisual programs and segments are stored. References from the Semantic Base and the TV-Anytime database to the corresponding programs and/or segments are maintained.

- The appropriate interfaces, which permit the end users to pose both simple keyword-based queries (according to TV-Anytime) and more powerful, semantic queries (supported by MPEG-7). These interfaces are based on the existence of appropriate query APIs (Application Programming Interfaces). The APIs supported validate their queries against the domain-specific ontologies and the application specific metadata before submitting the query. 


\section{OWL Ontology Integration Methodology}

In this section we describe the methodology that we have developed for the integration of OWL ontologies with the MPEG-7 and TV-Anytime standards. This integration is a cornerstone of the DS-MIRF framework for the support of ontology-based semantic indexing and retrieval of audiovisual content.

In the DS-MIRF framework semantic indexing produces consistent MPEG-7 and TV-Anytime compliant semantic descriptions for the audiovisual content. The indexing process is guided by appropriate domain-specific ontologies, which permit more accurate description of the concepts of each application domain than the generalpurpose concepts described by the standards themselves. In addition, ad-hoc domainspecific extensions of the standards are avoided. The domain-specific ontologies are based on the Semantic Part of the MPEG-7 MDS. Although it is possible to express the domain-specific ontologies using MPEG-7 syntax and structures [14] [17], interoperability issues make the use of the OWL ontology definition language more appealing, so the domain-specific ontologies in our framework are defined using the syntax of the OWL language. Thus, we have defined a core OWL ontology, which fully covers the Semantic Part of the MPEG-7 MDS, and then we define the domainspecific ontologies in OWL as extensions of the core ontology.

In the DS-MIRF framework we have adopted a two-layered model for multimedia metadata descriptions [15] [16]. The first layer is the metadata model defined in the Semantic Part of the MPEG-7 MDS, and the second layer is a set of domain-specific extensions of the first layer. The integration of OWL ontologies in the DS-MIRF framework provides a mechanism for capturing and extending the model for multimedia metadata descriptions using OWL ontologies and allows us to use these ontologies during semantic indexing and retrieval, remaining at the same time compatible with software which has been developed to work with the well-accepted standards of the audiovisual domain (TV-Anytime and MPEG-7).

The integration of OWL ontologies in the DS-MIRF framework utilizes three mechanisms:

a) A core OWL ontology, which fully covers the Semantic Part of the MPEG-7 MDS and captures the semantics of the first layer of the multimedia metadata model. We have decided to capture in the core ontology the MPEG-7 semantic metadata model, as it is more complete than the respective TV-Anytime model; in fact, the TV-Anytime model provides a subset of the semantic metadata representation facilities provided by the MPEG-7 model.

b) A methodology for the definition of domain-specific ontologies that extend the core ontology, in order to fully describe the concepts of specific application domains. The domain-specific ontologies defined using this methodology comprise the second layer of the multimedia metadata model.

c) Two sets of rules, used for the transformation of semantic metadata (formed according to the core ontology and its domain-specific extensions) to MPEG-7 and TV-Anytime compliant XML documents respectively. This way, interoperability with software developed to work with MPEG-7 and TV-Anytime is provided.

In the following sections we describe in more detail the above methodology and tools. The complete core ontology in OWL, as well as a complete example demonstrating the use of the methodology for domain-specific ontology definition in the domain of soccer games, are available at http://elikonas.ced.tuc.gr/ontologies/. 


\section{Core Ontology}

Our approach for the integration of OWL ontologies in the framework for the support of ontology-based semantic indexing and retrieval of audiovisual content, utilizes an ontology that captures the model provided by the Semantic Part of the MPEG-7 MDS for the representation of semantic metadata for audiovisual content description. This ontology captures the semantics of the first layer of the two-layered model for semantic metadata used in the DS-MIRF framework. The second layer of the model encapsulates domain-specific knowledge, which extends the audiovisual content description standards so that they integrate transparently domain-specific ontologies. This ontology, referred to as the Core Ontology in the rest of the paper, has been implemented in OWL and is described in this section.

The model for the semantic description of audiovisual content provided in the Semantic part of the MPEG-7 MDS is comprised of complex data types defined, using the XML Schema Language syntax [2], in a set of Description Schemes (DSs) rooted in the SemanticBase DS. The more important among the description schemes together with the complex data types defined in them are listed below:

1. SemanticBase DS: The abstract type SemanticBaseType is defined here. SemanticBaseType is the base type extended by other description schemes according to the needs for the description of semantic entities of specific types. SemanticBaseType has a set of simple attributes (id for instance identification, timeBase, timeUnit, mediaTimeBase and mediaTimeUnit for timing support) and the following complex attributes:

- AbstractionLevel, which represents the abstraction existing in the current semantic entity.

- Label, corresponding to a term that describes in brief the semantic entity.

- Definition, which is a textual annotation that describes the semantic entity.

- Property, which is a term that associates a property with the semantic entity.

- Relation, which relates the semantic entity with other semantic entities.

- MediaOccurrence, which relates the semantic entity to specific media items (e.g. video segments, images etc.).

2. SemanticBag DS and Semantic DS: Description schemes used for the description of collections of semantic entities. SemanticBagType is an abstract type, defined in the SemanticBag DS, which extends SemanticBaseType, while SemanticType is defined in the Semantic DS. SemanticType is a concrete type, thus its instances are used for the representation of semantic entity collections.

3. Object DS: The ObjectType defined here extends SemanticBaseType and is used for the description of objects and object abstractions (e.g. a table).

4. AgentObject DS: The actors that appear in an audiovisual segment are related with the instances of the AgentObjectType type that extends the ObjectType. Actors in general are represented using the AgentType, an abstract type extending SemanticBaseType defined in the Agent DS. PersonType, OrganizationType and PersonGroupType extend AgentType, are defined respectively in the Person DS, the Organization DS and the PersonGroup DS and are used for the representation of persons (e.g. football players), organizations and groups of persons. 
5. Event DS: The EventType defined here extends SemanticBaseType and is used for the description of events (e.g. a goal).

6. SemanticState DS: The SemanticStateType defined here extends SemanticBaseType and is used for the description of states described in an audiovisual segment and the parametric description of its features (e.g. the score in a soccer game before and after a goal).

7. SemanticPlace DS: The SemanticPlaceType defined here extends SemanticBaseType and is used for the description of places (e.g. Athens).

8. SemanticTime DS: The SemanticTimeType defined here extends SemanticBaseType and is used for the description of semantic time (e.g. Christmas).

9. Concept DS: The ConceptType defined here extends SemanticBaseType and is used for the description of concepts present in an audiovisual segment (e.g. cooperation).

10. SemanticRelation DS: The SemanticRelationType defined here extends SemanticBaseType and is used for the description of relationships among semantic entities. The relationships may be typed, as described in the SemanticRelation DS. In addition to the attributes inherited from SemanticBaseType, SemanticRelationType has the following attributes:

- Source, which is the id of the semantic entity that is the source of the relationship.

- Target, which is the id of the semantic entity that is the target of the relationship.

- Argument, which may be used as an alternate to source and target definition.

- Strength, which denotes the strength of the relationship.

- Name, which denotes the name of the relationship.

- Arity, which denotes the arity of the relationship.

- Properties, where the properties of the relationship are denoted.

For the representation of the Semantic part of MPEG-7 in OWL we developed the following methodology:

1. MPEG-7 Simple Datatype Representation: The simple datatypes needed are integrated in the core ontology, as OWL permits the integration in OWL ontologies of simple datatypes defined in the XML Schema Language [2].

2. MPEG-7 Complex Type Representation: MPEG-7 complex types correspond to OWL classes, which define groups of individuals that belong together because they share some properties. Thus, for every complex type defined in the MPEG-7 MDS a respective OWL class is defined.

2.1. Simple Attribute Representation: The simple attributes of the complex type of the MPEG-7 MDS are represented as OWL datatype properties, which relate class instances to datatype instances (e.g. integer, string etc.). Thus, for every simple attribute of the complex type a datatype property is defined.

2.2. Complex Attribute Representation: For the representation of the complex attributes of the complex type, OWL object properties are used, which relate class instances. For every complex attribute of the complex type the following actions are performed:

2.2.1. An OWL class for the representation of the complex attribute instances is defined, if it does not already exist. 
2.2.2. An OWL object property that relates the complex attribute instances with the appropriate complex type instances is defined.

2.3. Constraints: Constraints regarding value, cardinality and type for simple and complex attributes are expressed using the restriction mechanisms provided by OWL.

As an example, we show in Fig. 2 the definition of the OWL class "OrganizationType" (subclass of the "AgentType" that represents all the Agents), corresponding to the MDS complex type "OrganizationType". The "ElectronicAddress" object property, corresponding to the homonym complex attribute of the MDS "OrganizationType" type is also shown in Fig. 2.

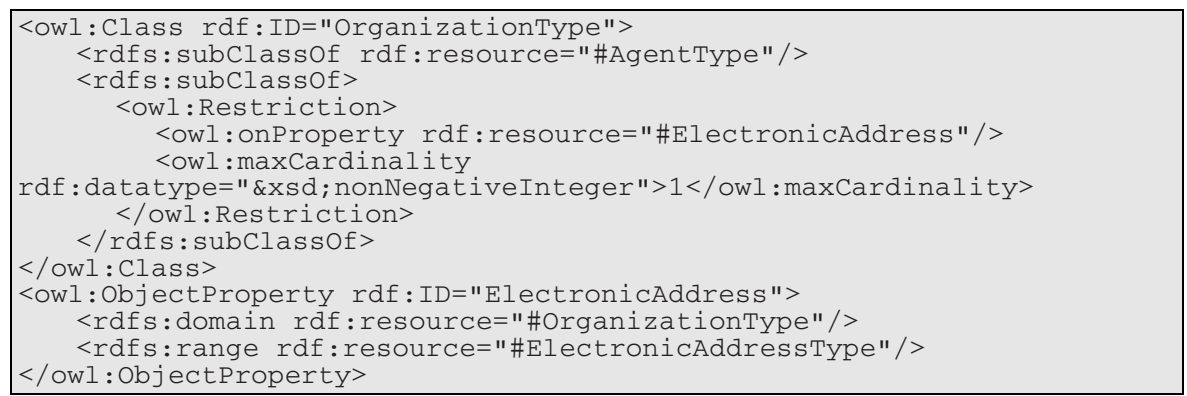

Fig. 2. OWL Definition of Organization

3. MPEG-7 Relationship Representation: Relationships between the OWL classes, which correspond to the complex MDS types, are represented by the instances of the "RelationBaseType" class and its subclasses. Every "RelationBaseType" instance is associated with a source and a target metadata item through the homonym object properties. A relationship is "attached" to a semantic entity through the "Relation" object property (corresponding to the "Relation" complex attribute of SemanticBaseType).

Relationships among semantic entities are represented by the instances of the "SemanticBaseRelationType" class (a "RelationBaseType" descendant) and the instances of its subclasses. The "SemanticBaseRelationType" subclasses, in addition to the other restrictions, have restrictions on the class that belongs their target and/or source. In Fig. 3 we show the definitions of the "RelationBaseType" class, its "source" datatype property and of the "ObjectObjectRelationType" class. The "ObjectObjectRelationType" class represents relationships between "ObjectType" instances and its source and target properties are restricted to have all their values from the "ObjectType" class instances.

The complete core ontology has been designed using the above rules but is not shown here due to space limitations. It is OWL-DL ontology, validated using the OWL species ontology validator ${ }^{1}$ and is available at http://elikonas.ced.tuc.gr/ontologies/av_semantics.zip.

1 The OWL species validator validates OWL ontologies and checks if an ontology conforms to one of the OWL species. It is available at http://phoebus.cs.man.ac.uk:9999/OWL/Validator 


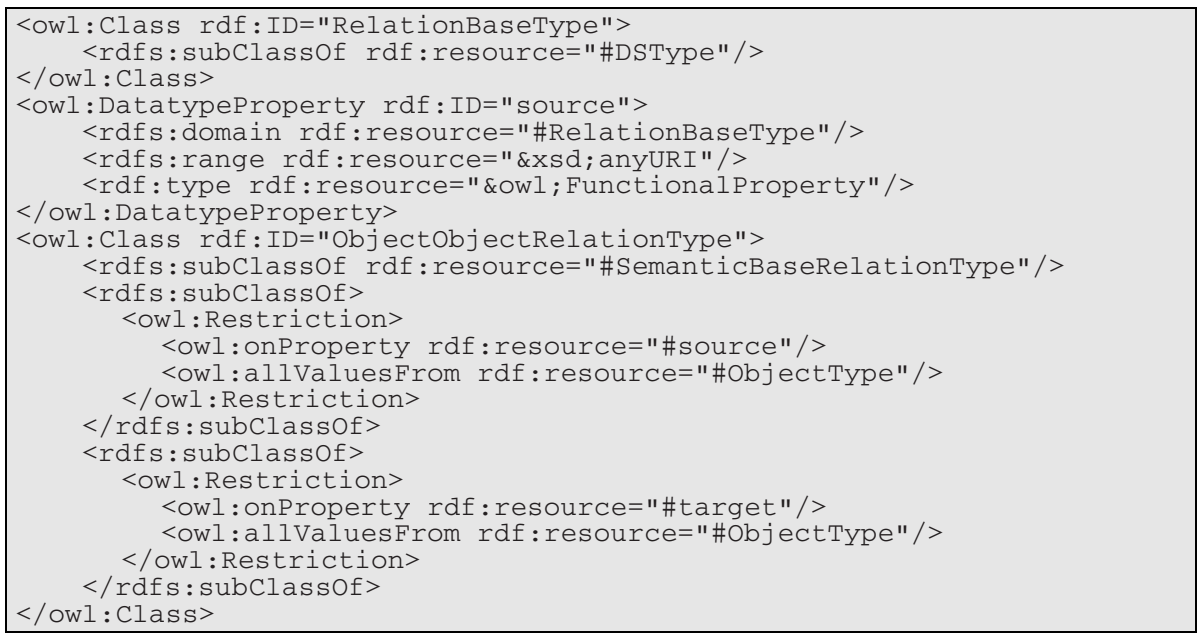

Fig. 3. OWL Definitions of RelationBaseType and ObjectObjectRelationType

\section{Methodology for the Definition of Domain-Specific Ontologies}

In this section we present the methodological steps for the definition of domainspecific ontologies and we make the definition process clear through a set of examples from different application domains.

As already mentioned in section 3, the domain-specific ontologies comprise the second layer of the semantic metadata model used in the DS-MIRF framework, with the first layer of the model encapsulated in the core ontology. Thus, the classes representing the domain-specific entities should be defined in a way that extends the core ontology. Having these in mind, the domain-specific ontologies are defined according to the following methodological steps:

1. Domain-specific entity types are represented by OWL classes defined to be subclasses of the appropriate core ontology classes. For example, in a football tournament application the "FootballTeam" subclass of the "OrganizationType" core ontology class, is used for the representation of football teams. As another example, in a Formula-1 championship application, the "Race" subclass of the "EventType" core ontology class will be used for race representation.

1.1. Attributes (simple or complex) that cannot be covered by the existing properties of the superclass are represented as appropriate object or datatype properties in order to make use of domain-specific knowledge (e.g. the shirt number of a football player can be represented as an integer datatype property).

1.2. On the attributes inherited from the parent class, some constraints may be applied using the restriction mechanism provided by OWL in order to make use of the domain knowledge and guide the indexers for the production of valid metadata (e.g. the "Place" property of the "EventType" class, that denotes the place where the event occurs, has to be restricted to Soccer Stadium values for football Match instances). 
As an example, we show in Fig. 4 the definition of the "FootballTeam" class, a subclass of the "OrganizationType" core ontology class, which represents football teams.

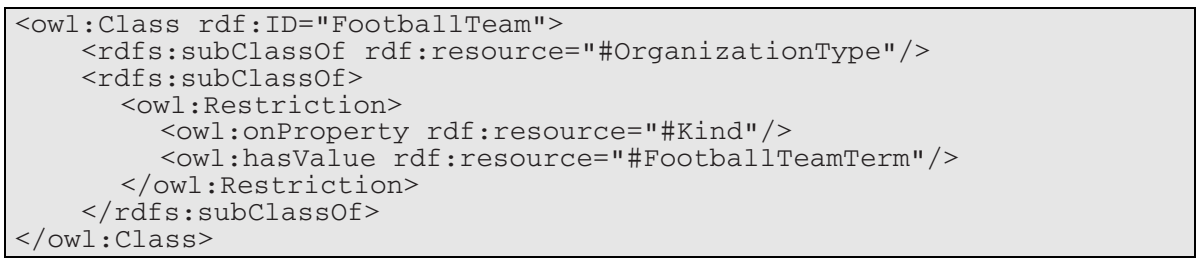

Fig. 4. OWL Definition of the FootballTeam class

2. Relationships with additional restrictions compared with the ones of the general relationships defined in the core ontology are usually needed (e.g. a Goal event may be related to player instances as goal agents). In these cases, the following actions are performed:

2.1. A subclass of "SemanticBaseRelationType" or of its appropriate subclass is defined and all the restrictions needed are set for the newly defined class.

2.2. An appropriate subproperty of the "Relation" property (inherited from the "SemanticBaseType" class) is defined in the domain of the classes capable of being sources of the relationship. We must note that OWL subproperties permit the implementation of property hierarchies: If an individual A is related to another individual $\mathrm{B}$ by property $\mathrm{C}$, which is a subproperty of property $\mathrm{D}$, then $\mathrm{A}$ is also related to $\mathrm{B}$ by $\mathrm{D}$.

For example, supposing that we would like to express the restriction that only players may be the agents of a Goal event, the following should be done:

- Definition of the "hasAgentOfPlayerRelationType" class (subclass of the "ObjectEventRelationType" that permits relating events with their agents). The "hasAgentOfPlayerRelationType" class must be restricted to have the "PlayerObject" class as target and the "Goal" class as permitted source.

- Definition of the "hasAgentOfPlayerRelation" object property (subproperty of the "Relation" property), having the "Goal" class as domain and as permitted range the "hasAgentOfPlayerRelationType" class.

In order to test the above-described methodology, we have developed a complete OWL (DL) ontology for football games, available at http://elikonas.ced.tuc.gr/ontologies/football.zip.

\section{Transformation Rules}

We describe in this section the transformation rules for the production of MPEG-7 and TV-Anytime compliant metadata from the RDF metadata structured according to the core ontology and the domain-specific ontologies and vice versa. The rules for the transformations to and from MPEG-7 are described in subsection 6.1, while the rules for the transformations to and from TV-Anytime are discussed in subsection 6.2. 


\subsection{Transformation to/from MPEG-7}

In this subsection we describe the rules for transforming into MPEG-7 compliant metadata the RDF metadata structured according to the OWL core ontology and the domain-specific ontologies developed according to the methodology discussed above.

We have already mentioned that MPEG-7 provides all the constructs needed for the structured description of audiovisual content. These constructs are the complex types defined in the MPEG-7 Description Schemes using the syntax of XML Schema language, so MPEG-7 metadata descriptions are XML documents.

Since MPEG-7 does not provide direct support for domain-specific ontologies, we automatically translate the entities of the supported domain-specific ontologies into abstract MPEG-7 descriptions using the "AbstractionLevel" attribute of the "SemanticBaseType" in order to form abstraction hierarchies. "AbstractionLevel" is a complex attribute of type "AbstractionLevelType" and has only one attribute, "Dimension", of non-negative integer type. When "AbstractionLevel" is not present in a semantic description, the description refers to specific audiovisual material. When "AbstractionLevel" is present, abstraction exists in the description. When "AbstractionLevel.Dimension" $=0$, there exists a description of a semantic entity (e.g. the football player Kahn) that is related to every audiovisual segment where the entity appears. When "AbstractionLevel" has a non-zero "Dimension", it can specify classes for the description of abstract semantic entities. The bigger the value of "AbstractionLevel.Dimension", the more extended the abstraction in the description (e.g. Kahn, which has "AbstractionLevel.Dimension"=0, is an instance of the "Goalkeeper" class, which has "AbstractionLevel.Dimension"=1, while "Goalkeeper" is an instance of the "Player" class, which has "AbstractionLevel.Dimension"=2). The reason for using this methodology (instead for example from subtyping) is that we remain this way completely compatible to MPEG-7 so that all tools and applications that use MPEG-7 still work transparently with the produced MPEG-7 metadata.

The rules for the transformation of OWL domain-specific ontologies into MPEG-7 compliant documents containing abstract descriptions are the following:

1. Every OWL class is transformed into an appropriate instance of the MPEG-7 class corresponding to its nearest ancestor in the core ontology.

1.1. The id of the instance is the OWL class name.

1.2. The type of the instance is the type of the MPEG-7 class corresponding to its nearest ancestor in the core ontology.

1.3. The "AbstractionLevel" of the instance is greater than or equal to 1 .

2. For every instance representing an OWL class, the following relationships are generated for the representation of subclass/superclass relationships:

2.1. A "specializationOf" relationship that relates the instance with the class or instance representing its direct superclass.

2.2. A set of "generalizationOf" relationships relating the instance with the instances representing its subclasses.

3. For each of the OWL class properties having a specific value in the abstract instance, the following take place:

3.1. If the property exists in its nearest ancestor in the core ontology, an instance of the corresponding MPEG-7 class attribute, having the desired value is generated. 
3.2. If the property is a subproperty of a property of the class nearest ancestor in the core ontology, an instance of the attribute of the MPEG-7 class corresponding to the ancestor having the desired value is generated.

3.3. If none of the above holds, an MPEG-7 "TermUseType" instance, holding the information needed for the representation of the value and the type of the property is generated. The generated "TermUseType" instance is a "Property" complex attribute of the instance ("Property" is an attribute inherited from "SemanticBaseType").

According to the above rules, the MPEG-7 abstract instance generated for the FootballTeam class (see Fig. 4) is shown in Fig. 5.

<Organization id="FootballTeam" xsi:type="OrganizationType">

$<$ AbstractionLevel dimension="1" />

$<$ Relation type="specializationof " target="OrganizationType" source="FootballTeam" />

$<$ Organization>

Fig. 5. MPEG-7 abstract description for the "FootballTeam" class

We must note here that OWL restrictions are not taken into account during the transformation process, as MPEG-7 does not support such restrictions for semantic entity instances (MPEG-7 supports restrictions at the schema level, in DS definition). For example, in the MPEG-7 description of the "FootballTeam" in Fig. 5, the restriction in "Kind" is not expressed. The restrictions are used during the semantic indexing process in order to enforce the indexers to define valid semantic metadata. This way, the produced MPEG-7 metadata are also valid.

Specific metadata descriptions are defined as specializations of the abstract entities, with "AbstractionLevel.Dimension=0", according to the following rules:

1. Every individual belonging to an OWL class defined in the core ontology is transformed into an appropriate instance of the corresponding MPEG-7 class.

1.1. For each of the individual's properties an instance of the corresponding MPEG-7 class attribute, which has the desired value, is generated.

1.2. The id of the instance is the individual's rdf:ID.

1.3. The type of the instance is the type of the corresponding MPEG-7 class.

2. Every individual belonging to an OWL class defined in a domain-specific ontology is transformed into an appropriate instance of the MPEG-7 class corresponding to the class nearest ancestor in the core ontology.

2.1. The id of the instance is the individual's rdf:ID.

2.2. The type of the instance is the type of the MPEG-7 class corresponding to the nearest ancestor in the core ontology of the class of the individual.

2.3. An "exampleOf" relation is generated, which relates the instance representing the individual with the instance representing the OWL class it belongs.

2.4. For each of the individual's properties, the same actions with the ones performed for an OWL class are performed.

For example, using the above rules, the generated MPEG-7 compliant description for the RDF description of the "Real" football team individual (see Fig. 6) that has been defined according to the football ontology, is shown in Fig. 7. 
$<$ FootballTeam rdf: ID="Real">

$<$ Name $>$ Real Madrid</Name>

$<$ haskind rdf:resource="\#FootballTeamTerm" / >

$</$ FootballTeam $>$

$<$ Term raf:ID="FootballTeamTerm"/>

Fig. 6. Definition of the "Real" football team

The individual "Real" defined in Fig. 6 belongs to the FootballTeam OWL class (which is subclass of the "OrganizationType" core ontology class). The team name is "Real Madrid" and is related with the "FootballTeamTerm" individual through the "Kind" property.

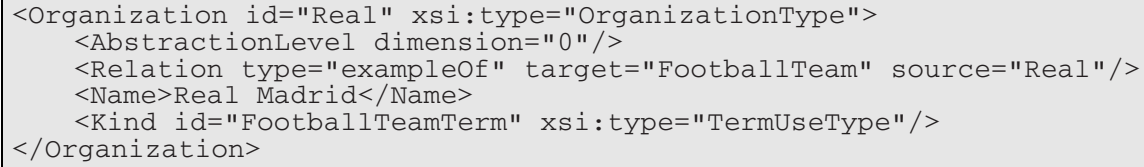

Fig. 7. MPEG-7 compliant metadata for the "Real" football team

The inverse process, for the transformation of MPEG-7 compliant metadata containing abstract object descriptions to OWL ontologies and the transformation of nonabstract MPEG-7 compliant metadata to RDF metadata structured according to the OWL ontologies is possible and of significant importance, because working with MPEG-7 is common in the multimedia community. The limitation in this case is that a part of the domain knowledge (e.g. restrictions) cannot be expressed in MPEG-7, so the produced domain-specific OWL ontologies and the corresponding RDF metadata will be less expressive.

\subsection{Transformation to/from TV-Anytime}

In this subsection we discuss the rules for transforming the RDF metadata structured according to the OWL core ontology and the domain-specific ontologies into TVAnytime compliant metadata.

As we have already mentioned, the only mechanism for the semantic description of audiovisual content provided by TV-Anytime is annotation based on keywords and keyword phrases. Thus, when translating RDF metadata defined using the OWL ontologies, we translate each metadata item to a set of keywords.

The following rules are applied, in order to obtain a TV-Anytime compliant keyword-based annotation from an RDF description structured according to the OWL core ontology and the domain-specific ontologies:

1. The name of the class to which an individual belongs, together with the rdf:ID of the individual, form a keyword phrase.

2. The name of each of the object properties of the individual is represented as a keyword.

2.1. The individuals related to the described individual through the object property are used for the production of keywords and keyword phrases after the keyword containing the name of the object property. 
3. A keyword phrase composed of the name and the value of every datatype property is produced.

3.1. A keyword phrase containing the value of the datatype property is produced.

In Fig. 8 we show the TV-Anytime compliant metadata produced for the "Real football team defined in Fig. 6 according to the above rules.

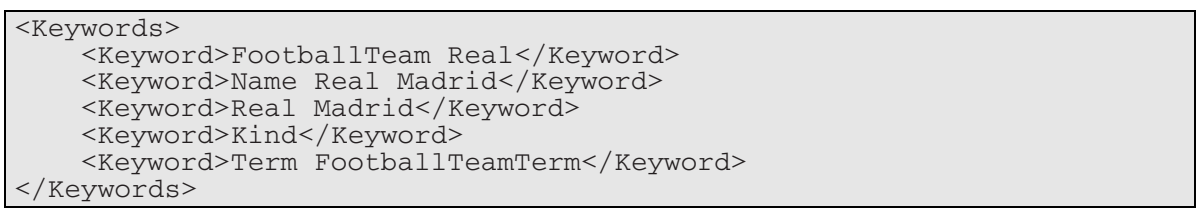

Fig. 8. TV-Anytime compliant metadata for the "Real" football team

As expected, the TV-Anytime compliant annotation can be exploited in a less flexible and efficient way than the equivalent MPEG-7 compliant one, since keywordbased descriptions have less expressive power than structured metadata descriptions.

This also implies that even if OWL ontology and RDF metadata production from the TV-Anytime compliant metadata is possible, it will give as a result ontologies where much of the domain knowledge is not precisely captured and incomplete RDF metadata descriptions.

\section{Conclusions - Future Work}

In this paper we have presented the integration of OWL ontologies in the DS-MIRF framework for the support of ontology-based semantic indexing and retrieval of audiovisual content following the MPEG-7 and TV-Anytime standard specifications for metadata descriptions. In this framework, semantic indexing produces consistent MPEG-7 and TV-Anytime compliant semantic descriptions for the audiovisual content. The indexing process is guided by appropriate domain-specific ontologies, which are based on the Semantic Part of the MPEG-7 MDS and are defined using the syntax of the OWL language. We have also presented an interoperability methodology and tools for transforming audiovisual content descriptions in MPEG-7 and TV-Anytime together with the domain-specific extensions in OWL and vice-versa.

The methodology of OWL ontology integration and the interoperability methodology and tools have been based on (a) A core OWL ontology, which fully covers the Semantic Part of the MPEG-7 MDS (b) A methodology for the definition of domainspecific ontologies that extend the core ontology in order to fully describe the concepts of specific application domains (c) Two sets of rules, used for the transformation of semantic metadata (formed according to the core ontology and its domainspecific extensions) to MPEG-7 and TV-Anytime compliant XML documents respectively.

The DS-MIRF framework for domain-specific multimedia information retrieval and filtering that includes the interoperability methodologies and tools presented in this paper has been implemented in the Linux OS environment. MySQL was the platform selected for the relational database (that contains both the Semantic Base and the 
TV-Anytime compliant database). XML data management in the relational database is carried out by a binder capable of fully representing XML documents as objects or objects as XML documents. The Segmentation \& Semantic Indexing Tool (including the module responsible for the translation between OWL/RDF, MPEG-7 and TVAnytime) has been implemented in java.swing.

Our future work in the domain of semantic description of audiovisual information includes:

- The extension of the core ontology, using the methodology discussed in section 3, in order to capture the other parts of the MPEG-7 metadata model (segmentation, user profiles, usage rights etc.).

- The deployment of the framework in order to provide automatic (or semiautomatic) abstractions of the audiovisual content, based on the domain-specific ontologies and the corresponding semantic information.

\section{Acknowledgments}

The work presented in this paper was partially funded in the scope of the DELOS II Network of Excellence in Digital Libraries (IST - Project Record Number 26059).

\section{References}

1. M.Doerr, J.Hunter, C.Lagoze, "Towards a Core Ontology for Information Integration", Journal of Digital Information, Volume 4 Issue 1, April 2003

2. D. Fallside, “XML Schema Part 0: Primer”, W3C Recommendation, 2001, http://www.w3.org/TR/xmlschema-0/

3. D. Mc Guiness, F. van Harmelen, "OWL Web Ontology Language Overview”, W3C Recommendation, 2004, http://www.w3.org/TR/owl-features/

4. J. Hunter, "Adding Multimedia to the Semantic Web - Building an MPEG-7 Ontology", in Proc. of International Semantic Web Working Symposium (SWWS), Stanford, July 30 August 1, 2001

5. J. Hunter, "Enhancing the Semantic Interoperability of Multimedia through a Core Ontology", IEEE Transactions on Circuits and Systems for Video Technology, Special Issue on Conceptual and Dynamical Aspects of Multimedia Content Description, Feb 2003

6. ISO/IEC JTC 1/SC 29/WG 11/N3966, "Text of 15938-5 FCD Information Technology Multimedia Content Description Interface - Part 5 Multimedia Description Schemes", Singapore, 2001

7. A. Jaimes, T. Echigo, M. Teraguchi, F. Satoh, "Learning Personalized Video Highlights from Detailed MPEG-7 Metadata", in Proc. of ICIP, Rochester, NY, USA, 2002

8. F. de Jong, T. Westerveld, "MUMIS: Multimedia Indexing and Searching", in Proc. of CBMI Workshop, Brescia, Italy, 2001, pp. 423-425

9. F. Kazasis, N. Moumoutzis, N. Pappas, "Informative Annex: Generic Architecture for handling TVA Metadata using relational database technologies", contribution TV-Anytime Forum/Metadata WG, $2^{\text {nd }}$ Implementer's workshop, Geneva, 2002

10. G. Klyne, J. Caroll, "Resource Description Framework (RDF): Concepts and Abstract Syntax", W3C Recommendation, 2004, http://www.w3.org/TR/rdf-concepts/ 
11. MPEG Group, "MPEG-7 (Multimedia Content Description Interface)", http://www.chiariglione.org/mpeg/index.htm

12. TV-Anytime Forum, http://www.tv-anytime.org/

13. R. Troncy, "Integrating Structure and Semantics into Audio-visual Documents", in Proc. of $2^{\text {nd }}$ International Semantic Web Conference (ISWC), 20-23 October 2003, Sanibel Island, Florida, USA

14. C. Tsinaraki, E. Fatourou, S. Christodoulakis, "An Ontology-Driven Framework for the Management of Semantic Metadata describing Audiovisual Information", in Proc. of CAiSE, Velden, Austria, 2003, pp 340-356

15. C. Tsinaraki, S. Papadomanolakis, S. Christodoulakis, "A Video Metadata Model supporting Personalization \& Recommendation in Video-based Services", in Proc. of MDDE Workshop (in conjunction with RETIS), Lyon, France, 2001, pp. 104-109

16. C. Tsinaraki, S. Papadomanolakis, S. Christodoulakis, "Towards a two - layered Video Metadata Model”, in Proc. of DEXA Workshop - DLib, Munich, Germany, 2001, pp 937941

17. C. Tsinaraki, P. Polydoros, F. Kazasis, S. Christodoulakis, "Ontology-based Semantic Indexing for MPEG-7 and TV-Anytime Audiovisual Content", Special issue of Multimedia Tools and Applications Journal on Video Segmentation for Semantic Annotation and Transcoding, 2004 